\title{
Malformación cavernosa de la región pineal. Caso clínico y revisión de la literatura
}

\author{
C. Chamadoira; A. Cerejo; A. Vilarinho; L. Castro* y R. Vaz
}

Departamentos de Neurocirugía y de Anatomía Patológica*. Hospital de São João. Porto. Portugal.

\section{Resumen}

Las malformaciones cavernosas de la región pineal son raras, con solamente 21 casos publicados hasta el momento en la literatura. A pesar de la dificultad de su diagnóstico debido a su extrema rareza, la presencia de esta lesión puede ser sospechada basándonos en sus características radiológicas.

Presentamos el caso de una paciente de 57 años de edad, con un cuadro clínico de instauración aguda de desorientación, somnolencia y diplopia. La TC cerebral mostró una hemorragia aguda en la región pineal e hidrocefalia triventricular asociada y la RM cerebral fue sugestiva de que se tratase de una malformación cavernosa. La paciente fue operada con escisión total de la lesión y el estudio histológico confirmó el diagnóstico de angioma cavernoso. Concluimos que la cirugía para extracción total de la lesión es el tratamiento de elección cuando, basados en la imagen, sospechamos un angioma cavernoso de la región pineal.

PALABRAS CLAVE: Angioma cavernoso. Región pineal. Resonancia magnética.

Cavernous malformation of the pineal region. Case report and review of the literature

\section{Summary}

Cavernous malformations rarely occur in the pineal region with only 21 reported to date. Although its diagnosis is not easy because of the extreme rareness of this condition, the presence of this lesion can be suspected based on its typical radiological findings.

We report the case of a 57-year-old woman presented with desorientation, somnolence and diplopy. The CT-scan showed an acute hemorrhage in the pineal region and triventricular hydrocephalus. An MRI suggested a cavernous malformation. The

Recibido: 11-09-09. Aceptado: 5-11-09 patient was operated with total en-bloc removal of the lesion. We conclude that surgical exploration and total resection is the treatment of choice when the diagnosis of cavernous angioma is suspected of the basis of neuroimaging.

KEY-WORDS: Cavernous angioma. Magnetic resonance. Pineal region

\section{Introducción}

Las malformaciones cavernosas son lesiones bien conocidas entre los hamartomas vasculares del sistema nervioso central, estando descritas a lo largo de todo el neuroeje, siendo más frecuentes en el tronco cerebral o subcorticales cerca de la cisura interhemisférica y los ganglios de la base ${ }^{1,13}$. Sin embargo, la región pineal es una localización rara para este tipo de lesiones. El primer caso de angioma cavernoso de la región pineal fue descrito en el año 1961 por Miller ${ }^{17}$. Desde entonces han sido publicados otros 20 casos de angiomas cavernosos con esta localización $n^{4-6,8,10,13-19,25-28}$. Poco se ha escrito sobre las características diagnósticas de estas lesiones cuando están localizadas en esta región, siendo con alguna frecuencia confundidos con otros tumores de la región pineal, sobre todo con tumores de células germinales ${ }^{14}$. Los angiomas cavernosos presentan una apariencia reticular típica en resonancia magnética, con una zona central de intensidades mixtas y un anillo hipointenso en T2 que, sin embargo, puede variar dependiendo del tiempo de evolución de las hemorragias ${ }^{1}$.

Presentamos un nuevo caso de angioma cavernoso de la región pineal tratado quirúrgicamente y realizamos una revisión de la literatura.

\section{Caso Clínico}

Una mujer de 57 años de edad, sin antecedentes patológicos de interés, fue admitida en el Servicio de Urgencias de nuestro hospital con un cuadro agudo de cefaleas, somnolencia, desorientación y diplopia. El examen neurológico 


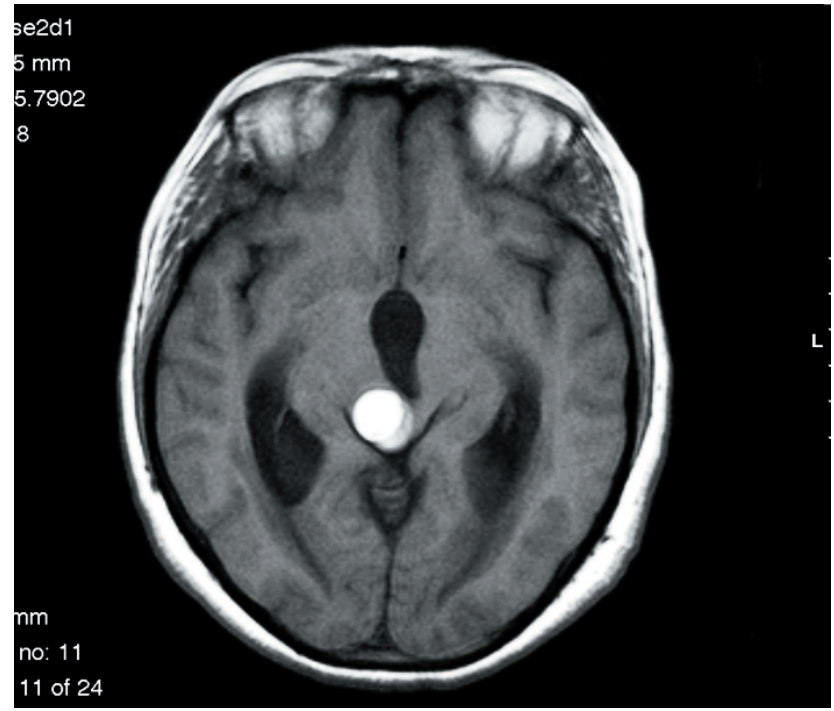

Figura 1. $R M$ realizada tras la aparición de los síntomas (A): T1-axial mostrando una lesión hiperintensa, redondeada en la región pineal e hidrocefalia asociada.

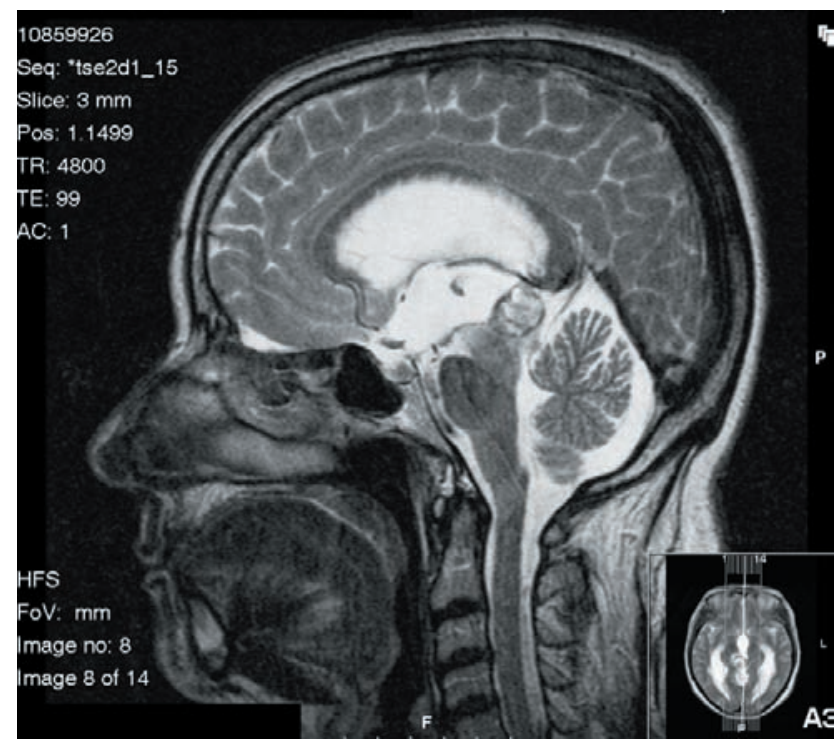

Figura 1. (B): T2-sagital que muestra un anillo hipointenso en la periferia de la lesión.

mostró una limitación de la supraversión (Signo de Parinaud) y edema de papila bilateral. El resto del examen neurológico era normal.

La TC cerebral mostró una hemorragia en la región pineal e hidrocefalia triventricular asociada. La RM cerebral reveló una lesión redondeada, de contornos bien definidos, localizada en la región pineal. Esta lesión era hiperintensa en la secuencia $\mathrm{T} 1$, hiper/iso intensa en la secuencia T2, con un anillo hipointenso perilesional e iso/ hiperintensa con un anillo hipointenso en la secuencia T2Flair. La lesión condicionaba compresión del Acueducto de Silvio con hidrocefalia triventricular asociada (Fig. 1). El

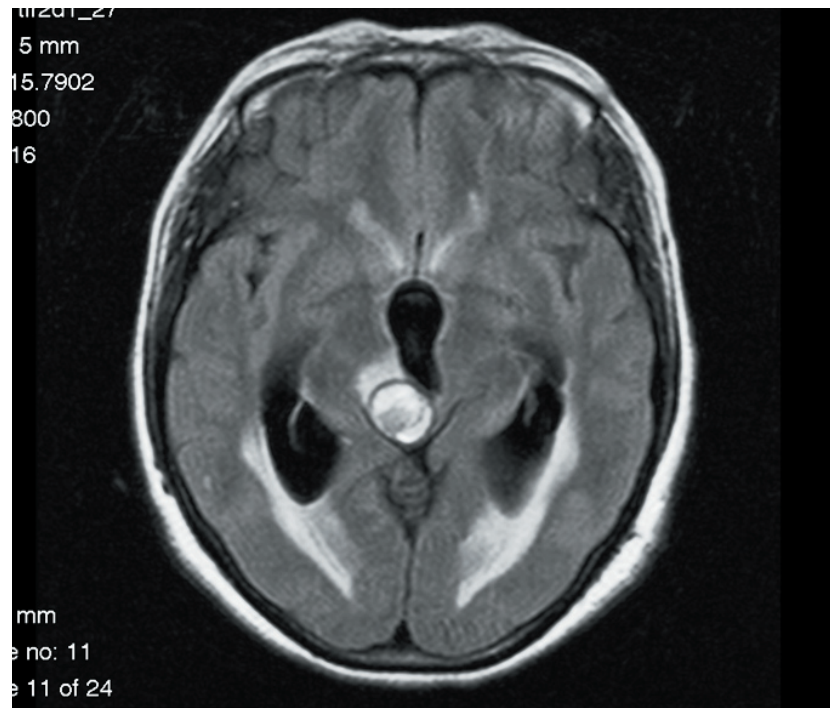

Figura 1. (C): T2-Flair axial que muestra la lesión hiper/ isointensa con un anillo hipointenso completo en su periferia.

diagnóstico diferencial para esta lesión incluía un tumor de células germinales, una lesión neoplásica del parénquima de la glándula pineal, un tumor de serie glial o una malformación vascular.

Para excluir una malformación vascular se realizó una angiografía digital, no siendo encontrada ninguna anomalía vascular. Los niveles de $\alpha$-fetoproteína ( $\alpha$-FP), antígeno carcinoembrionario (CEA) y gonadotrofina coriónica humana $(\beta-\mathrm{HCG})$ fueron negativos en sangre y líquido cefalorraquídeo. No fueron identificadas células tumorales en el líquido cefalorraquídeo.

Debido a la ausencia de un diagnóstico histológico, al tamaño de la hemorragia y a la persistencia de los síntomas, fue decidido tratamiento quirúrgico.

La paciente fue operada en posición de semisentado, con un acceso supracerebeloso infratentorial, siendo observadas loculaciones hemorrágicas y una lesión rojiza, de consistencia fibrosa y bien circunscrita. Esta lesión fue fácilmente disecada del tejido circundante, que incluía el mesencéfalo, así como de bandas de aracnoides en torno a la vena de Galeno. La lesión fue extraída en su totalidad.

No hubo complicaciones significativas durante el postoperatorio y la paciente se recuperó progresivamente de las alteraciones visuales. La histopatología de la lesión reveló una malformación cavernosa con capilares alargados de finas paredes de colágeno cubiertas por una única capa de epitelio y rodeada por hematomas en diferentes fases. No fueron encontradas células tumorales (Fig. 2).

Seis meses después del procedimiento la paciente había regresado a sus actividades habituales y tenía un examen neurológico normal. La RM cerebral de control mostró una 


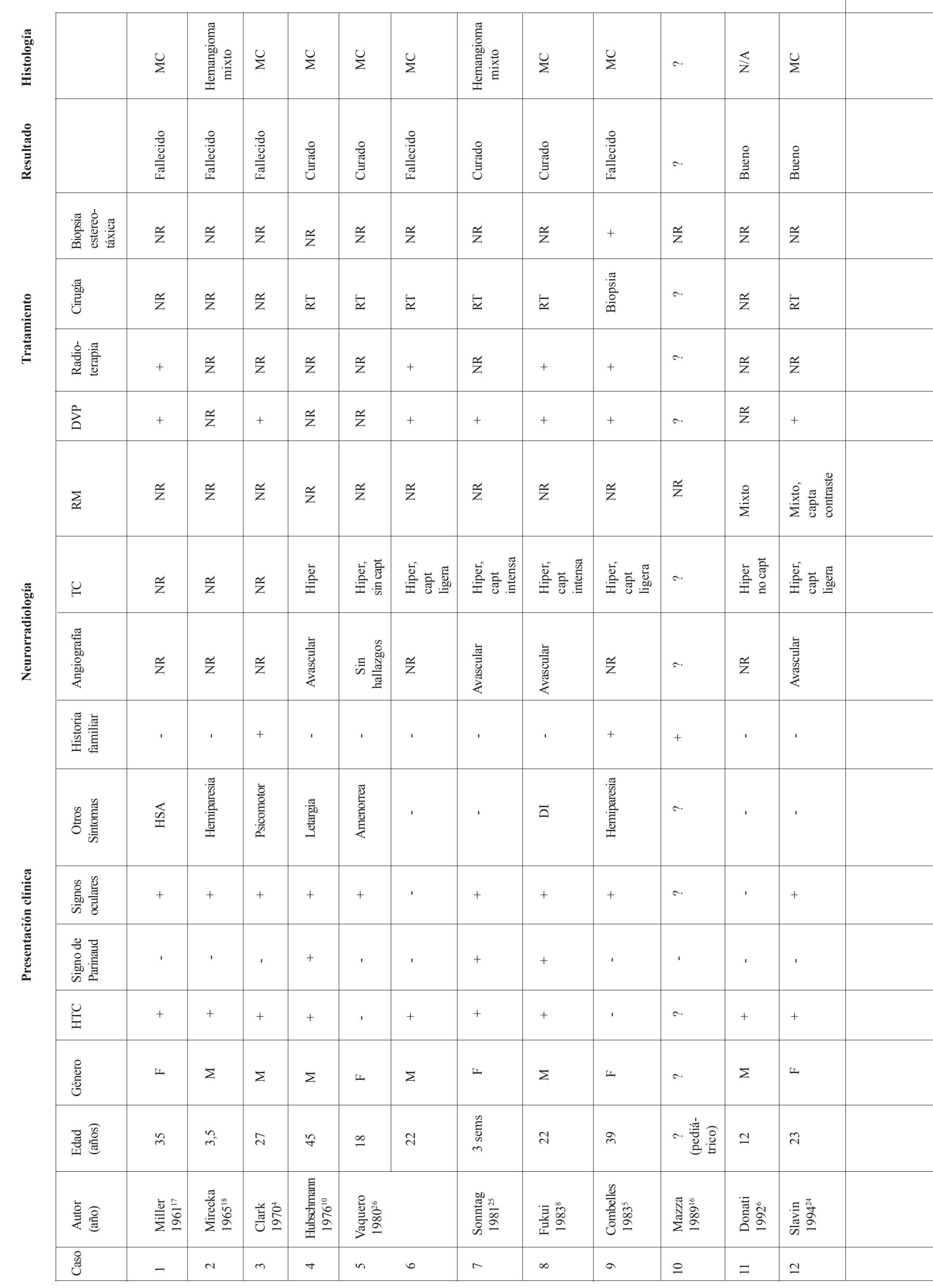




\begin{tabular}{|c|c|c|c|c|c|c|c|c|c|c|}
\hline$\stackrel{\circlearrowright}{\Sigma}$ & $\stackrel{U}{\Sigma}$ & $\overleftrightarrow{\mathrm{Z}}$ & $\stackrel{U}{\Sigma}$ & $\stackrel{\circlearrowright}{\Sigma}$ & $\sum_{\Sigma}^{U}$ & $\stackrel{U}{\Sigma}$ & $\sum_{\Sigma}^{U}$ & $U_{\Sigma}$ & $\sum_{\Sigma}^{U}$ & 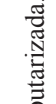 \\
\hline 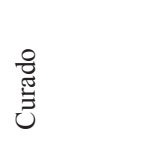 & 苞 & $\frac{8}{\frac{\pi}{\pi}}$ & 莡 & 莺 & 莺 & $\frac{\stackrel{8}{\pi}}{\frac{\pi}{\pi}}$ & 莺 & 莺 & $\begin{array}{l}\stackrel{8}{\pi} \\
\text { Jँ }\end{array}$ & 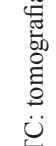 \\
\hline 占 & 号 & 兑 & 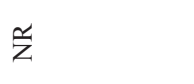 & + & 号 & 号 & 兑 & 艺 & 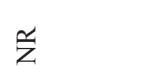 & : \\
\hline 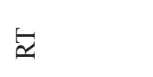 & 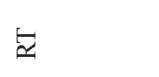 & 岂 & $\underline{\widetilde{q}}$ & $\underline{\sim}$ & $\underline{\mathfrak{q}}$ & $\stackrel{\leftarrow}{\simeq}$ & $\bar{\simeq}$ & $\underline{\underline{a}}$ & $\overrightarrow{\underline{a}}$ & 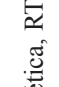 \\
\hline 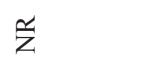 & 艺 & 兑 & 㟔 & 岂 & 占 & 㟔 & 点 & 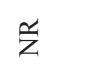 & 兑 & 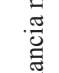 \\
\hline 岂 & 岂 & + & + & + & ' & + & + & + & 岂 & 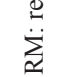 \\
\hline 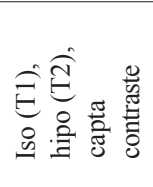 & 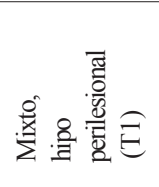 & 离总总高 & 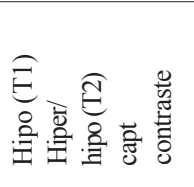 & 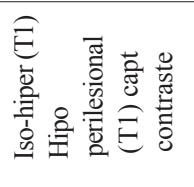 & 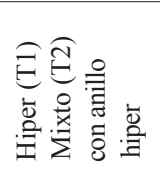 & $\tilde{z}$ & 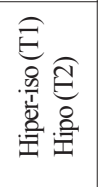 & $\sum_{\substack{x \\
x^{*}}}$ & 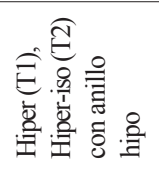 & 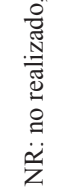 \\
\hline 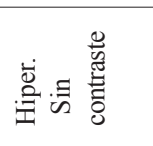 & 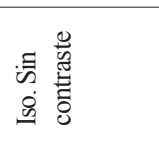 & 苛咅 & 占 & 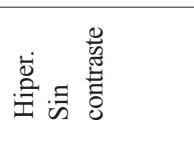 & 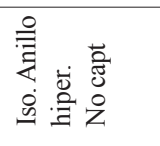 & 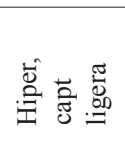 & 离 & $\stackrel{\overline{0}}{:}$ & 芯 & 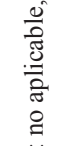 \\
\hline 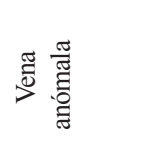 & 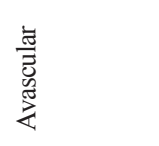 & 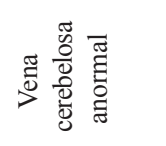 & 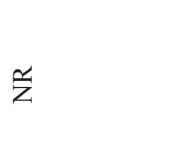 & 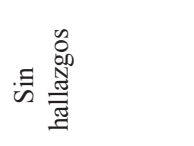 & 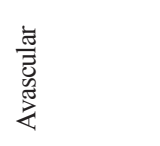 & 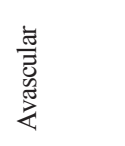 & 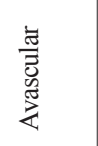 & 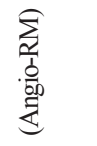 & 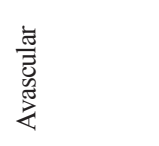 & . \\
\hline ' & ' & ' & ' & ' & 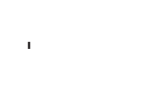 & ' & ' & , 总 & ' & 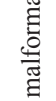 \\
\hline ' & ' & ' & & 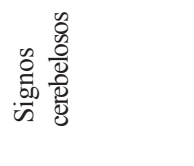 & 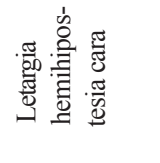 & ' & ' & & ' & 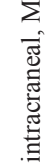 \\
\hline+ & + & + & + & + & + & + & + & + & + & 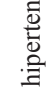 \\
\hline+ & ' & + & + & + & + & ' & + & , & + & $\begin{array}{l}E \\
\text { IJ }\end{array}$ \\
\hline+ & ' & + & + & + & + & + & + & + & + & 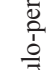 \\
\hline I & ⿷匚 & L & 山 & $\Sigma$ & I & 山 & I & L & I & 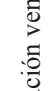 \\
\hline 2 & $\stackrel{\infty}{n}$ & $\cong$ & $\bar{m}$ & 'f & $=$ & in & F & $\hat{n}$ & in & ¿̆. \\
\hline 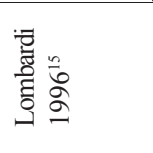 & & & 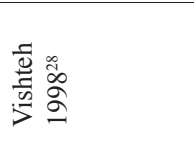 & 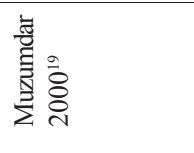 & 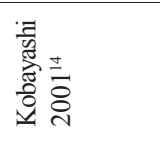 & 要 & 寻 & & 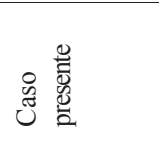 & 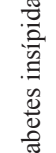 \\
\hline 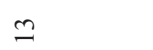 & \pm & $\cong$ & $\stackrel{0}{\circ}$ & $=$ & $\stackrel{\infty}{\sim}$ & 2 & ¿ి & $\vec{\sim}$ & $\tilde{A}$ & $\ddot{\vec{\Delta}}$ \\
\hline
\end{tabular}




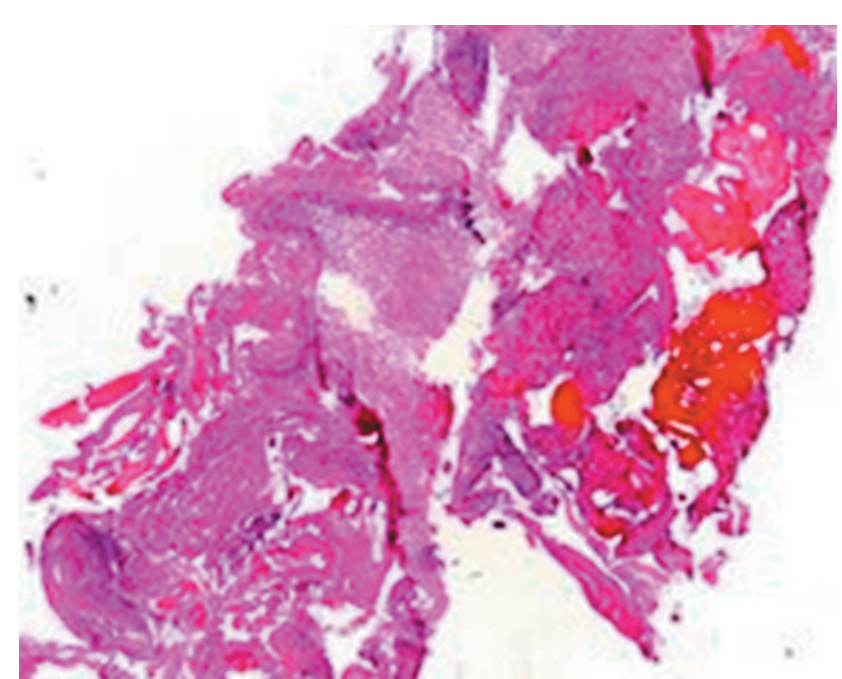

Figura 2. El examen histopatológico (hematoxilina-eosina) muestra un aglomerado de vasos de distintos calibres y pared fina rodeados por hemorragias en diferentes estadios y macrófagos.

resección completa de la lesión así como resolución de la hidrocefalia (Fig. 3).

\section{Discusión}

La región pineal es una localización rara para los angiomas cavernosos. El primer caso de angioma cavernoso de la región pineal fue descrito en el año 1961 por Miller, en el que la lesión fue parcialmente extraída. La paciente acabó por fallecer debido a sucesivas hemorragias subaracnoideas ${ }^{17}$. Desde entonces han sido publicados otros 20 casos de angiomas cavernosos de la región pineal de los cuales 17 fueron confirmados después del estudio histológico. La prevalencia de estas lesiones aumenta en la segunda y tercera décadas de vida ${ }^{15,25}$. El paciente más joven en que fue diagnosticado un angioma cavernoso de la región pineal tenía 3 semanas de vida, y el mayor 73 años de edad. Es más común en el género femenino siendo que, 13 de los 21 casos eran mujeres. En 3 casos fue encontrada una historia familiar de angiomas cavernosos ${ }^{4,5,16}$. Se sabe que la ocurrencia familiar sigue un patrón de herencia autosómico dominante, con penetrancia variable ${ }^{1}$. Nuestra paciente no presentaba antecedentes familiares de malformaciones vasculares.

No existen características clínicas que nos permitan realizar el diagnóstico de esta lesión, ya que los síntomas y signos de presentación son idénticos a los de otras lesiones de la región pineal. Dieciocho pacientes se presentaron con clínica de hipertensión intracraneal ${ }^{4,6,8,10,13,15,17-19,24-28}$

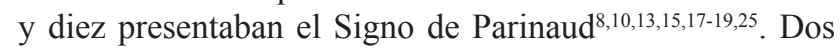
pacientes presentaban hemiparesia ${ }^{5,18}$, uno hemihipostesia de la cara $^{24}$, y otro síntomas cerebelosos ${ }^{19}$. En un caso se

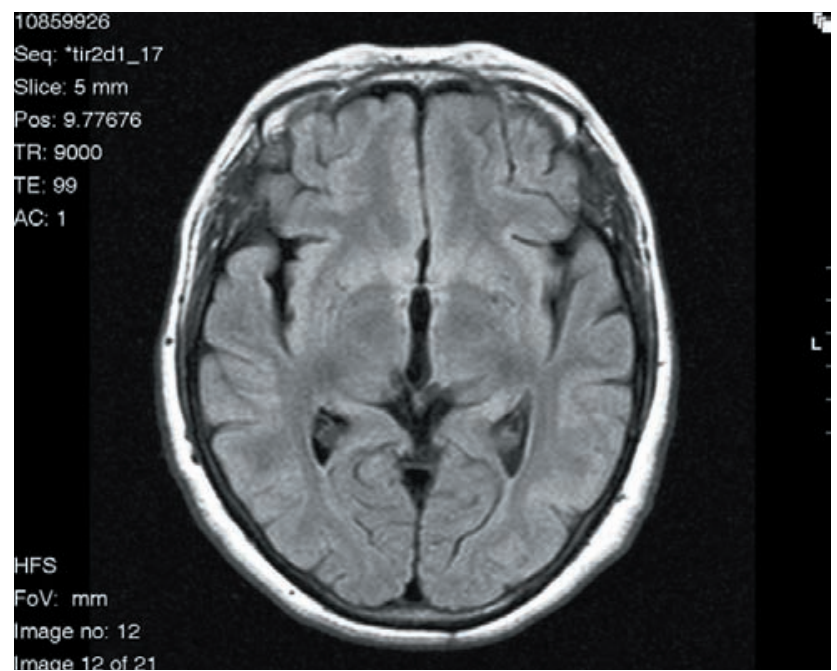

Figura 3. RM de control (6 meses post-op): (A) T2-Flair axial.

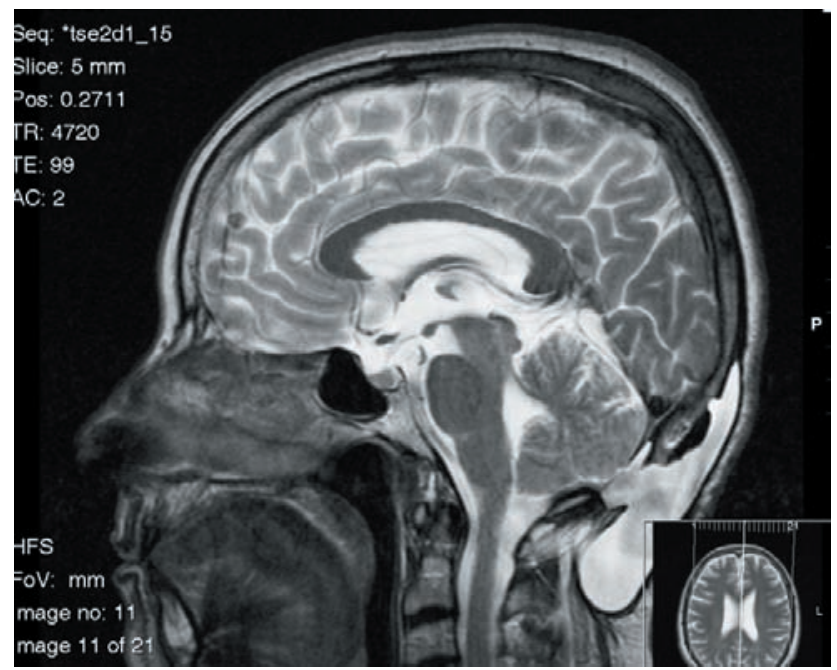

Figura. 3. (B): T2-sagital muestra la resección completa de la lesión y la resolución de la hidrocefalia.

verificó diabetes insípida y en otro, amenorrea con niveles de prolactina aumentados ${ }^{8,26}$. Estos fenómenos son probablemente consecuencia de la distensión del suelo del tercer ventrículo que puede dañar al hipotálamo, derivando en alteraciones endocrinológicas que incluyen atrofia de los genitales, obesidad, pubertad precoz y diabetes insípida.

Hasta la introducción de la resonancia magnética, el diagnóstico de esta lesiones era retrospectivo, basado en el estudio histológico. Sin embargo, las características de imagen de los angiomas cavernosos son lo suficientemente típicas como para permitir su diagnóstico en la gran mayoría de casos. En los casos de hemorragia aguda, la lesión será isointensa o ligeramente hipointensa y marcadamente hipointensa respectivamente en las secuencias T1 y T2. En las hemorragias subagudas tardías, el área central será hiperintensa en las secuencias T1 y T2. En las lesiones 
asociadas a hemorragias crónicas, encontraremos una señal hipointensa en $\mathrm{T} 1$ e hiperintensa con un anillo periférico hipointenso en T2. La captación de contraste puede sugerir la presencia de venas de desarrollo anómalo asociadas a la malformación ${ }^{1}$.

De los 21 casos publicados en la literatura, diez realizaron RM. Únicamente en nuestro caso, está especificada la presencia del característico anillo hipointenso en la secuencia T2.

La angiografía es normal en el $30-40 \%$ de los pacientes, y raramente las malformaciones cavernosas son identificadas directamente con la angiografia ${ }^{1}$. El valor de esta técnica en los angiomas cavernosos es en primer lugar el reconocimiento de malformaciones vasculares, sobre todo venas anómalas, una vez que en el procedimiento quirúrgico se pretende la extracción de la malformación cavernosa preservando la vena de desarrollo anómalo. Once pacientes realizaron angiografía, y en dos se encontró una vena anómala asociada ${ }^{15}$. Un paciente realizó Angio-RM, que mostró una lesión avascular ${ }^{13}$.

En 13 de los 21 pacientes, se implantó una válvula ventrículo-peritoneal, previamente al tratamiento definitivo $4,5,8,13,15,17,24-28$. En cuatro pacientes fue aplicada radioterapia ${ }^{5,8,17,26}$. Únicamente en un caso se realizó una biopsia estereotáxica ${ }^{5}$. En 14 casos se consiguió una resección total de la lesión ${ }^{8,10,13,15,19,24-28}$. Cinco pacientes murieron ${ }^{4,5,10,17,18,26}$ y diez pacientes obtuvieron un resultado excelente después de la extracción total de la lesión ${ }^{8,10,13-15,19,25,26,28}$.

El diagnóstico diferencial de los angiomas cavernosos de la región pineal debe establecerse principalmente con malformaciones arterio-venosas (MAV,s) crípticas -parcialmente trombosadas- $\mathrm{y}$ con lesiones neoplásicas ${ }^{1-3,7,9,11,20,22,23}$. Las malformaciones arterio-venosas presentan una señal de flujo serpinginoso característico, arterias dilatadas y venas de drenaje. Las hemorragias asociadas a neoplasias suelen presentar un edema circundante significativo, el efecto de masa suele ser mayor y el anillo hipointenso circundante puede ser incompleto debido al depósito inconsistente de hemosiderina. El coriocarcinoma y las metástasis de melanoma deben ser sospechadas en el caso de hemorragia reciente ${ }^{7}$.

En 18 de los 21 casos publicados en la literatura, el diagnóstico de angioma cavernoso fue confirmado por estudio histológico. Estas lesiones se caracterizan por no tener parénquima entre los canales vasculares. La lesión esta rodeada por un grado variable de gliosis. Inflamación, calcificaciones e incluso osificación pueden estar presentes, especialmente en lesiones grandes. Los canales vasculares pueden estar trombosados, presentando varios estadios de reorganización. La vascularización circundante suele ser normal; la nutrición arterial raramente se visualiza ${ }^{1}$.

El tratamiento de los tumores de la región pineal ha generado, y todavía genera, ciertas controversias. Con frecuencia estos pacientes eran sometidos a la colocación de derivaciones y posteriormente realizaban radioterapia. Esto era debido a la dificultad de un diagnóstico preoperatorio fiable y a que un porcentaje elevado de neoplasias de la pineal son radiosensibles. Sin embargo, no todas lo son. La biopsia estereotáxica ha sido preconizada por algunos autores como alternativa a la cirugía previamente a la aplicación de terapias complementarias. Sin embargo, este procedimiento es potencialmente peligroso debido a la naturaleza vascular de los angiomas cavernosos y a su frecuente asociación con venas de desarrollo anómalo. El papel de la radiocirugía en el tratamiento de los angiomas cavernosos permanece en discusión. La radiocirugía estereotáxica ha sido preconizada para el tratamiento de angiomas cavernosos pequeños en áreas críticas. Una disminución significativa de la tasa de sangrado se ha encontrado a partir de los 3 años del tratamiento ${ }^{1}$. Estudios a largo plazo y con un cuidadoso seguimiento clínico y de imagen son necesarios para establecer el verdadero papel de la radiocirugía en el tratamiento de esta entidad.

La resonancia magnética es muy sensible en el diagnóstico de los angiomas cavernosos y esto ha favorecido la resección microquirúrgica de estas lesiones en lugar de la radioterapia o la biopsia estereotáxica. El papel de la cirugía en el tratamiento de los angiomas cavernosos es también controvertido. Los angiomas cavernosos asintomáticos no requieren, en principio, tratamiento profiláctico. Esto es debido a que la morbilidad y mortalidad asociadas a hemorragias originadas en estas lesiones, es relativamente baja $^{12,21}$. Sin embargo, publicaciones más recientes recomiendan el tratamiento quirúrgico en el caso de hemorragias clínicamente significativas, epilepsia de difícil control o deterioro neurológico. Además, una vez que se ha producido un primer sangrado, la tasa de resangrado es relativamente alta ${ }^{12}$. Como se observa en nuestro caso, una malformación cavernosa de la región pineal tiene asociado al riesgo de sangrado, la posibilidad de provocar hidrocefalia obstructiva por compresión del acueducto.

\section{Conclusiones}

Las hemorragias de la región pineal son raras y pueden tener su origen en diversas entidades entre las que se encuentran tumores y lesiones vasculares, incluyendo los angiomas cavernosos. A pesar de que el diagnóstico de las malformaciones cavernosas de esta localización no es fácil dada su extrema rareza, su presencia debe ser sospechada debido a sus hallazgos típicos en RM. Si no existen dudas en el diagnóstico, la escisión total de la lesión es el tratamiento de elección en pacientes jóvenes y sanos debido al riesgo de hemorragias recurrentes, hidrocefalia y progresivo agravamiento neurológico. El tratamiento quirúrgico con la resección total de la lesión ofrece la curación para este tipo de lesiones. 


\section{Bibliografía}

1. Awad, I.A., Barrow, D.L.: Cavernous Malformations; AANS Publications Committee, 1993.

2. Braun, V., Antoniadis, G., Rath, S., Richter, H.P.: Cavernoma. Indications for surgical removal and outcome. Nervenarzt 1996; 67: 301-305.

3. Burres, K.P., Hamilton, R.D.: Pineal apoplexy. Neurosurgery 1979; 4: 264-268.

4. Clark, J.V.: Familial occurrence of cavernous angiomata of the brain. J Neurol Neurosurg Psychiatry 1970; 33: 871876.

5. Combelles, R.J., Blond, S., Biondi, A., CombellesPruvot, M., Szikla, G., Christiaens, J.L.: Familial forms of intracranial cavernous hemangioma. Neurochirurgie 1983; 29: 263-269.

6. Donati, P., Maiuri, F., Gangemi, M., Gallicio, B., Sigona, L.: Cavernous angioma of the pineal region. J Neurosurg Sci 1992; 36: 155-160.

7. Fujii, T., Itakura, T., Hayashi, S., Komai, N., Nakamine, H., Saito, K.: Primary pineal choriocarcinoma with hemorrhage monitored by computerized tomography. Case report. J Neurosurg 1981; 55: 484-487.

8. Fukui, M., Matsuoka, S., Hasuo, K., Numaguchi, Y., Kitamura, K.: Cavernous hemangioma in the pineal region. Surg Neurol 1983; 20: 209-215.

9. Harada, K., Hayashi, T., Anegawa, S., et al.: Pineocytoma with intratumoral hemorrhage following ventriculoperitoneal shunt. Neurol Med Chir (Tokyo) 1993; 33: 836-838.

10. Hubschmann, O., Kasoff, S., Doniger, D., Llena, J., Leed, N.: Cavernous hemangioma in pineal region. Surg Neurol 1976; 6: 349-351.

11. Kawakami, Y., Yamada, O., Tabuchi, K., Ohmoto, T., Nishimoto, A.: Primary intracranial choriocarcinoma. J Neurosurg 1980; 53: 369-374.

12. Kim, D.S., Park, Y.G., Choi, J.U., Chung, S.S., Lee, K.C.: An analysis of the natural history of cavernous malformations. Surg Neurol 1997; 48: 9-18.

13. Kim, D.S., Shim, K.W., Kim, T.G., Chang, J.H., Park, Y.G., Choi, J.U.: Pineal cavernous malformations: report of two cases. Yonsei Med J 2005; 46: 851-858.

14. Kobayashi, S., Kamagata, M., Nakamura M., Nakazato, Y., Sasaki, T.: Pineal apoplexy due to massive hemorrhage associated with cavernous angioma: case report. Surg Neurol 2001; 55: 365-371.

15. Lombardi, D., Scheithauer, B.W., Villani, R.M., Giovanelli, M., de Tribolet, N.: Cavernous haemangioma of the pineal region. Acta Neurochir (Wien) 1996; 138: 678-683.

16. Mazza, C., Scienza, R., Dalla Bernardin, B., Beltramello, A., Bontempini, L., Dapian, R.: Cerebral cavernous mal- formations (cavernomas) in children. Neurochirurgie 1989; 35: 106-108.

17. Miller, R.H.: Spontaneous subarachnoid hemorrhage: A presenting symptom of a tumor of the third ventricle. Surg Clin North Am 1961; 41: 1043-1048.

18. Mirecka, K.: A case of an angiomatous tumor localised near the pineal body of a child. Pol Przegl Radiol Med Nukl 1965; 20: 307-311.

19. Muzumdar, D.P., Misra, B.K., Bhaduri, A.S.: Pineal region cavernoma-case report. Neurol Med Chir (Tokyo) 2000; 40: 372-379.

20. Olson, J.R., Abell, M.R.: Haemangiopericytoma of the pineal body. J Neurol Neurosurg Psychiatry 1969; 32: 445449.

21. Porter, P.J., Willinsky, R.A., Harper, W., Wallace, M.C.: Cerebral cavernous malformations: natural history and prognosis after clinical deterioration with or without hemorrhage. J Neurosurg 1997; 87: 190-197.

22. Razario, R., Adelman, L., Prager, R.J., Stein, B.M.: Meningiomas of the pineal region and third ventricle. Neurosurgery $1979 ; 5$ : 489-495.

23. Ruiz Sandoval, J.L., Cantu, C., Barinagarrementeria, F.: Intracerebral hemorrhage in young people: analysis of risk factors, location, causes, and prognosis. Stroke 1990; 30: 537541.

24. Slavin, K.V., Dujovny, M., McDonald, L.W., Camras, L.R., Ausman, J.I.: Pineal region: rare location of a cavernous haemangioma. Neurol Res 1994; 16: 133-136.

25. Sonntag, V.K.H., Waggener, J.D., Kaplan, A.M.: Surgical removal of a hemangioma of the pineal region in a 4-weekold infant. Neurosurgery 1981; 8: 568-588.

26. Vaquero, J., Carrillo, R., Cabezudo, J., Leunda, G., Villoria, F., Bravo, G.: Cavernous angiomas of the pineal region. Report of two cases. J Neurosurg 1980; 53: 833-835.

27. Vhora, S., Kobayashi, S., Okudera, H.: Pineal cavernous angioma presenting with Parkisonism. J Clin Neurosci 2001; 8: 263-266.

28. Vishteh, A.G., Nadkarni, T., Spetzler, R.F.: Cavernous malformation of the pineal region: short report and review of the literature. Br J Neurosurg 2000; 14: 147-151.

Chamadoira, C.; Cerejo, A.; Vilarinho, A.; Castro, L.; Vaz, R.: Malformación cavernosa de la región pineal. Caso clínico y revisión de la literatura. Neurocirugía 2010; 21 : 138-145.

Correspondencia: Clara Chamadoira Villaverde. Neurosurgery Department. Hospital de São João. Alameda Professor Hernâni Monteiro, 4200-319, Porto. Portugal.

E-mail: clarachamadoira@gmail.com 
Comentario al trabajo Malformación cavernosa de la región pineal. Caso clínico y revisión de la literatura de Chamadoira y cols.

Los autores nos presentan el caso de una paciente con una lesión hemorrágica de la región pineal e hidrocefalia obstructiva en la que un único procedimiento, la exéresis quirúrgica precoz, fue resolutiva.

En primer lugar, se trata de un caso interesante por la localización excepcional de una de estas malformaciones vasculares en esta región. Como apuntan los autores, sólo existen 21 casos de angiomas cavernosos de la región pineal descritos previamente en la literatura.

En segundo lugar, por el diagnóstico diferencial preoperatorio que plantea una masa pineal hemorrágica, que algunos autores denominan apoplejía pineal. Las lesiones pineales que más frecuentemente causan hemorragia son los tumores de células germinales, sobre todo los coriocarcinomas, aunque también algunos tumores de células pineales y algunos quistes pineales pueden debutar de esta forma. En este sentido, los niveles de $\alpha$-FP y $\beta$-HCG en sangre y LCR y los hallazgos en la RM pueden orientar al diagnóstico.

En tercer lugar, creemos que la realización de arteriografía cerebral es estos casos puede no ser necesaria, desde la amplia disponibilidad y cada vez mayor resolución del angio-TC y la angio-RM. Estas dos pruebas no invasivas probablemente tengan un alto valor predictivo a la hora de descartar una MAV como causa del sangrado y para dibujar el árbol vascular, sobre todo venoso, de cara a la planificación quirúrgica.

En cuarto lugar, nos parece que la actitud terapéutica elegida por los autores, la exéresis quirúrgica completa precoz a través de una vía supracerebelosa infratentorial, es el tratamiento de elección en los casos en que se sospecha un cavernoma, ya que con un solo procedimiento -aunque no exento de riesgos- se puede lograr la curación del paciente, se consigue muestra para su análisis anatomopatológico y se elimina la causa de la hidrocefalia. Pensamos que este planteamiento es más efectivo que el tratar primero la hidrocefalia, ya sea con drenaje ventricular externo, derivación ventrículo-peritoneal o ventriculostomía endoscópica, y en un segundo tiempo operar la lesión, aunque esta opción podría ser válida en casos en los que la hidrocefalia produzca un deterioro neurológico rápido y el neurocirujano no esté familiarizado con la vía de abordaje a la región pineal. Una tercera forma de tratamiento sería tratar en primer lugar la hidrocefalia y posteriormente vigilar o radiar la lesión ya sea con radioterapia convencional o con radiocirugía. Este último planteamiento mas conservador, ampliamente utilizado en el pasado, evita los riesgos de un abordaje a la región pineal y resuelve los síntomas producidos por la hidrocefalia, pero no los producidos por el efecto masa local ni aporta evidencia anatomopatológica de la naturaleza de la lesión subyacente, pudiendo tener su papel en el paciente mayor, o con patología sistémica concomitante, que lo hicieran mal candidato a la cirugía ${ }^{1}$.

\section{Bibliografía}

1. Avery, G.J., Lind, C.R., Bok, A.P.: Succesful conservative operative management of pineal apoplexy. Clin Neurosci 2004; 11: 667-669.

A. de la Lama Zaragoza Vigo 